\title{
Factores que inciden en la deserción escolar
}

Factors that affect school dropout

\author{
Luis R. Ruiz-Ramírez a, Ma. de Lourdes E. García-Vargas ${ }^{b}$,Héctor D. Molina-Ruiz ${ }^{c}$, Gerardo \\ Reyes-Ruiz ${ }^{d}$
}

\begin{abstract}
:
This research aims to determine the factors that affect school dropout in the industrial engineering (IE) career of the Autonomous University of the State of Hidalgo, Tepeji del Río Higher School. To do this, a validated measuring instrument was generated with the Cronbach Alpha tool, a 5-point Likert scale was used, through a pilot survey that allowed the sample to be calculated. 58 questionnaires were applied in the 9 semesters of the race. The reasons identified were: engineering students are studying and working at the same time and sometimes opt for work, there is a lack of motivation in subjects by teachers, students do not enter classes, there is no coherence in the topics taught and those identified in the curriculum. In this way, this research was able to determine the factors influencing the dropout of 208 (IE) students, so that authorities can make relevant decisions to reduce dropout rates. The studio has the ability to support top-level school authorities to recognize the possible causes of dropout they have in their schools and for the community that requires guidance from this article.
\end{abstract}

Keywords:

Desertion, possible causes, investigation.

Resumen:

La presente investigación tiene como objetivo determinar los factores que inciden en la deserción escolar en la carrera de Ingeniería Industrial de la Universidad Autónoma del Estado de Hidalgo, Escuela Superior de Tepeji del Río. Para ello, se generó un instrumento de medición validado con la herramienta Alfa de Cronbach, se utilizó una escala Likert de 5 puntos, a través de una encuesta piloto que permitió calcular la muestra. Se aplicaron 58 cuestionarios en los 9 semestres de la carrera. Las causas que se identificaron fueron las siguientes: los estudiantes de ingeniería están estudiando y trabajando al mismo tiempo y a veces optan por el trabajo, existe una falta de motivación en las asignaturas por parte de los profesores, los estudiantes no entran a clases, no hay coherencia en los temas enseñados y los que se identifican en la currícula. De esta manera, la investigación fue capaz de determinar los factores que influyen en la deserción de 208 estudiantes de la (IE), para que las autoridades puedan tomar decisiones pertinentes para reducir las tasas de deserción escolar. El estudio tiene capacidad para apoyar a las autoridades escolares de nivel superior para reconocer las posibles causas de deserción que tienen en las escuelas y para la comunidad que requiere la guía de este artículo.

\section{Palabras Clave:}

Deserción, causas posibles, investigación

\section{Introducción}

La Escuela Superior Tepeji del Río (ESTeR) se fundó como parte del proceso de descentralización de los programas educativos de la Universidad Autónoma del Estado de Hidalgo (UAEH), a fin de atender las demandas regionales y dar respuesta a las necesidades económicas, sociales y educativas de la región. Inició actividades el 30 de septiembre del 2002, abrió sus aulas a un total de 40 alumnos en la Licenciatura en Ingeniería Industrial (LII), esta generación egresó en diciembre del 2006, con un índice de retención de $22.5 \%$ [1].

El estudio se justifica ya que en la ESTeR en la (LII) la tasa de deserción es alarmante, ya que ha estado presente en todos los semestres e incluso en algunos periodos ha aumentado, desde el inicio de labores en el año 2002 segundo periodo hasta fecha. La permanencia del estudiante en la carrera de ingeniería sigue siendo un problema que no

${ }^{a}$ Universidad Autónoma del Estado de Hidalgo, https://orcid.org/0000-0003-3299-214X, Email: ru271343@uaeh.edu.mx. 
se ha erradicado, en los últimos cinco años se han identificado diferentes variables que influyen para que los estudiantes dejen sus estudios sin terminar, sería un error generalizar los diferentes estudios, por lo que la relevancia del presente estudio se enfoca al orientar para describir el comportamiento en cuanto al abandono escolar. La pregunta de investigación será ¿Cuáles son las causas que inciden significativamente en la deserción de los estudiantes de la Escuela Superior de Tepeji del Rio de la Licenciatura en Ingeniería Industrial?

El Objetivo general del estudio es determinar las causas que inciden significativamente en la deserción de los estudiantes de la Escuela Superior de Tepeji del Rio de la Licenciatura en Ingeniería Industrial en la cohorte 2015 segundo periodo al 2018 segundo periodo (1502-1802).

Estudios sobre deserción escolar. Un estudio realizado en 2017 por la OCDE (Organización para la cooperación y desarrollo económicos) [2] solo 10 adultos mayores de 25 años nacidos en México han terminado su educación universitaria.

Las posibles causad de acuerdo a la revista Edurama [3] son: carencia en la habilidad de aprendizaje, la falta de asesoría y apoyo en el momento de tomar decisiones académicas. El número de desempleados titulados, poca inversión pública del estado mexicano en comparación con países como Canadá, Suiza y en Latinoamérica en países como Brasil y Chile.

Según datos del INEGI (Instituto Nacional de Estadística Geografía e Informática) solo ocho de cada 100 alumnos que inician estudios universitarios los concluyen. El INEGI argumenta que los principales factores de deserción a nivel universitario son el poco interés en el estudio y cuestiones económicas [4].

Para corroborar la presencia y en aumento de los índices de deserción y retención se realizó una búsqueda en la página oficial de la (UAEH), en el módulo de información para el estudio de trayectorias escolares [5], se observa que, en la generación 2002 segundo periodo, ingresaron cuarenta alumnos de los cuales solamente concluyeron nueve, generando así un índice de deserción elevado y alarmante del $77.5 \%$. Así como se presentó un índice de deserción para esta generación, de igual manera se presenta en todas y cada una de ellas en los últimos cinco años. La información se encuentra en la tabla 1.

Tabla 1. Indicas de ingresos, retención y deserción escolar de septiembre 2002 -diciembre 2004.

\begin{tabular}{|c|c|c|c|c|c|}
\hline $\begin{array}{c}\text { Generacion } \\
\text { es }\end{array}$ & Ingre & Retenci & Deserci & Índice de & Índice de \\
sos & ón & ón & deserción & retención \\
\hline 0202 & 40 & 9 & 31 & $77.50 \%$ & $22.50 \%$ \\
\hline 0301 & 18 & 7 & 11 & $61.11 \%$ & $38.89 \%$ \\
\hline 0302 & 44 & 16 & 28 & $63.64 \%$ & $36.36 \%$ \\
\hline 0401 & 16 & 3 & 13 & $81.25 \%$ & $18.75 \%$ \\
\hline 0402 & 31 & 12 & 19 & $61.29 \%$ & $38.71 \%$ \\
\hline & & & & Promedio & $31.04 \%$ \\
\hline
\end{tabular}

Se observa que los índices de deserción son altos y variables en las cinco generaciones estudiadas, se revisa que los ingresos en las generaciones par son mayores que en las generaciones non y que el índice de retención es bajo.

Se estudiaron cuantitativamente las cohortes que van desde el año 2015 a 2018, en cada uno de ellos se puede observar toda la población que ingresa a la licenciatura y se puede apreciar que en todos ellos por lo menos durante el primer semestre cuentan con una matrícula efectiva. En cada uno de las cohortes se observó que del primero al segundo semestre el porcentaje de descensión es alarmante ya que presenta un equivalente al $29 \%$ presentado en la (tabla 2), el cual quiere decir que es seguro que deserten el $29 \%$ de la matricula que ingresa en la carrera, se analizaron generaciones anteriores donde se pudieron ver cifras alarmantes en la generación 2013 segundo periodo (1302) fue la de mayor índice de deserción del primero al segundo semestre con un $54.3 \%$.

Tabla 2. Índices de deserción escolar presentes por generación

\begin{tabular}{|c|c|c|c|}
\hline Generación & $\begin{array}{c}\text { Matricula 1 } \\
\text { Semestre }\end{array}$ & $\begin{array}{c}\text { Matricula 2 } \\
\text { Semestre }\end{array}$ & $\begin{array}{c}\text { Índice de } \\
\text { deserción }\end{array}$ \\
\hline 1502 & 30 & 24 & $20.0 \%$ \\
\hline 1601 & 15 & 12 & $20.0 \%$ \\
\hline 1602 & 46 & 37 & $19.6 \%$ \\
\hline 1701 & 16 & 9 & $43.8 \%$ \\
\hline 1702 & 29 & 18 & $37.9 \%$ \\
\hline 1801 & 23 & 13 & $43.5 \%$ \\
\hline 1802 & 63 & 52 & $17.5 \%$ \\
\hline 1901 & 18 & 18 & $0.0 \%$ \\
\hline & & Promedio & $29 \%$ \\
\hline
\end{tabular}

El problema no termina en estos periodos si no que el mismo fenómeno se va presentando, pero en menor proporción hasta concluido el 6 semestre, es en el cambio de ciclo escolar donde las matrículas ya se conservan estables hasta concluir el 9 semestre.

Finalmente, se logró precisar un porcentaje de deserción de la generación 2002 segundo periodo (0202) a la generación 2015 primer periodo (1501) del 58\% (ver tabla 3 y gráfica 1 ), y 53\% para la matricula que se tiene de la generación 1502 hasta la cohorte de la generación 2018 segundo periodo (1802) mostrado en la tabla 5; cabe mencionar que estas últimas generaciones son de la matricula inscrita en la (LII) de la cohorte 2015 segundo periodo a 2019 primer periodo (1502-1901) y que la mayoría de esta matricula hoy en día aún se encuentra estudiando la licenciatura.

Tabla 3. Índices de deserción escolar 2002-2015.

\begin{tabular}{|c|c|c|c|c|}
\hline $\begin{array}{c}\text { Generacione } \\
\text { s }\end{array}$ & $\begin{array}{c}\text { Ingre } \\
\text { sos }\end{array}$ & $\begin{array}{c}\text { Egresa } \\
\text { dos }\end{array}$ & $\begin{array}{c}\text { Deserci } \\
\text { ón }\end{array}$ & $\begin{array}{c}\% \text { De } \\
\text { Deserción }\end{array}$ \\
\hline 0202 & 40 & 9 & 31 & $78 \%$ \\
\hline
\end{tabular}




\begin{tabular}{|c|c|c|c|c|}
\hline 0301 & 18 & 7 & 11 & $61 \%$ \\
\hline 0302 & 44 & 16 & 28 & $64 \%$ \\
\hline 0401 & 16 & 3 & 13 & $81 \%$ \\
\hline 0402 & 31 & 12 & 19 & $61 \%$ \\
\hline 0501 & 7 & 3 & 4 & $57 \%$ \\
\hline 0502 & 16 & 12 & 4 & $25 \%$ \\
\hline 0602 & 25 & 8 & 17 & $68 \%$ \\
\hline 0701 & 4 & 2 & 2 & $50 \%$ \\
\hline 0702 & 29 & 16 & 13 & $45 \%$ \\
\hline 0801 & 15 & 6 & 9 & $60 \%$ \\
\hline 0802 & 41 & 21 & 20 & $49 \%$ \\
\hline 0901 & 14 & 4 & 10 & $71 \%$ \\
\hline 0902 & 30 & 14 & 16 & $53 \%$ \\
\hline 1001 & 15 & 7 & 8 & $53 \%$ \\
\hline 1002 & 24 & 11 & 13 & $54 \%$ \\
\hline 1101 & 15 & 5 & 10 & $67 \%$ \\
\hline 1102 & 32 & 24 & 8 & $25 \%$ \\
\hline 1201 & 24 & 9 & 15 & $63 \%$ \\
\hline 1202 & 39 & 9 & 30 & $77 \%$ \\
\hline 1301 & 9 & 5 & 4 & $44 \%$ \\
\hline 1302 & 35 & 11 & 24 & $69 \%$ \\
\hline 1401 & 15 & 6 & 9 & $60 \%$ \\
\hline 1402 & 32 & 16 & 16 & $50 \%$ \\
\hline 1501 & 14 & 4 & 10 & $71 \%$ \\
\hline & & & $\begin{array}{c}\text { Promed } \\
\text { io }\end{array}$ & $58 \%$ \\
\hline
\end{tabular}

Grafica 1. Índices de ingresos, retención y deserción escolar

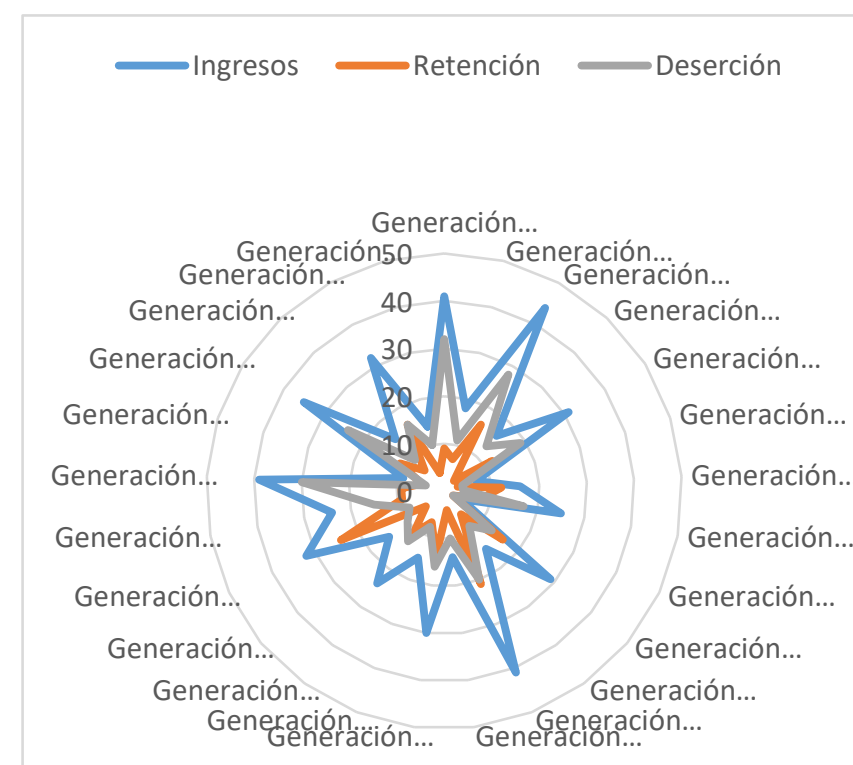

A continuación, se presenta una tabla que señala los índices de deserción generaciones 2015-2019
Tabla 4. Índices de deserción escolar de la Licenciatura en Ingeniería Industrial en la Escuela Superior Tepeji generaciones 2015-2019.

\begin{tabular}{|c|c|c|c|c|}
\hline $\begin{array}{c}\text { Generacio } \\
\text { nes }\end{array}$ & $\begin{array}{c}\text { Ingres } \\
\text { os }\end{array}$ & $\begin{array}{c}\text { Retenci } \\
\text { ón }\end{array}$ & $\begin{array}{c}\text { Deserci } \\
\text { ón }\end{array}$ & $\begin{array}{c}\% \text { De } \\
\text { Deserción }\end{array}$ \\
\hline 1502 & 30 & 9 & 21 & $70 \%$ \\
\hline 1601 & 15 & 6 & 9 & $60 \%$ \\
\hline 1602 & 46 & 21 & 25 & $54 \%$ \\
\hline 1701 & 16 & 6 & 10 & $63 \%$ \\
\hline 1702 & 29 & 14 & 15 & $52 \%$ \\
\hline 1801 & 23 & 10 & 13 & $57 \%$ \\
\hline 1802 & 63 & 52 & 11 & $17 \%$ \\
\hline 1901 & 18 & 18 & 0 & $0 \%$ \\
\hline & & & $\begin{array}{c}\text { Promed } \\
\text { io }\end{array}$ & $53 \%$ \\
\hline & & & & \\
\hline
\end{tabular}

\section{Metodología}

La metodología para el estudio tuvo un enfoque cuantitativo, se diseñó y aplico una encuesta de opinión dirigida a los estudiantes de todos los semestres de la (UAEH) en la (ESTeR) de la (LII). Para el formato de la encuesta se construyeron 27 reactivos, para definir las opciones de respuesta se consideró la escala Likert de 5 puntos la cual expresa, cuando así sea el caso, su grado de acuerdodesacuerdo (5 totalmente de acuerdo y 1 totalmente en desacuerdo) la cual es adecuada para el estudio en pie. Para aplicar las encuestas se expresó a los participantes que sus respuestas eran confidenciales y solo para fines de investigación.

La herramienta utilizada para verificar la fiabilidad del instrumento de evaluación fue utilizar el Alfa de Cronbach[6], el cual permitió observar que el instrumento utilizado para la integración de datos tiene correspondencia con los objetivos de la investigación previamente establecidos obteniendo un Alfa de Cronbach basado en elementos estandarizados de 0.757 (tabla 5), esto muestra que el instrumento de evaluación presenta una correlación positiva alta, y que toda la información que se recabe a partir de la aplicación de las encuestas tiene un grado de fiabilidad adecuado para determinar las causas de deserción que se están buscando.

Tabla 5. Alfa de Cronbach calculado con SPSS

\begin{tabular}{|c|c|c|}
\hline $\begin{array}{c}\text { Alfa de } \\
\text { Cronbach }\end{array}$ & $\begin{array}{c}\text { Alfa de Cronbach } \\
\text { basada en elementos } \\
\text { estandarizados }\end{array}$ & $\begin{array}{c}\text { N de } \\
\text { elementos }\end{array}$ \\
\hline .656 & .757 & 27 \\
\hline
\end{tabular}

Cálculo del tamaño de la muestra. Para determinar el tamaño de la muestra se consideraron varios aspectos, relacionados con tamaño de la población, el nivel de confianza, la probabilidad de éxito o proporción esperada, probabilidad de fracaso y el error máximo admisible en términos de proporción [7]. Realizando el análisis estadístico correspondiente a una matrícula de 208 estudiantes mediante un proceso de muestreo no probabilístico de tipo 
estratificado, se obtuvo el número de estudiantes que debían ser considerados para tener una muestra representativa de la población. Este análisis se llevó a cabo utilizando la siguiente formula:

$$
\begin{gathered}
n=\frac{208 *(1.96)^{2} * 70 * 30}{(10)^{2} *(208-1)+(1.96)^{2} * 70 * 30}=58.3 \\
=58 \text { Muestras }
\end{gathered}
$$

Se estableció el nivel de confianza en 95\% y el error estándar del $10 \%$, de acuerdo a datos sugeridos de diferentes autores; cabe mencionar que la probabilidad de éxito del $70 \%$ que se tomó está en base a la fiabilidad que tiene el cuestionario antes calculada. Considerando estos valores y aplicando la ecuación, se tiene que la muestra necesaria, para ser considerada como representativa de la población estudiada, es de al menos 58 alumnos pertenecientes a los 9 semestres de la matricula actual de la licenciatura de Ingeniería Industrial.

El objetivo del cuestionario es capturar con un nivel de detalle avanzado, las posibles causas de deserción escolar de los estudiantes de nivel superior.

Aplicación de la encuesta. Una vez teniendo información acerca de los índices de deserción, se optó por aplicar una encuesta a 58 estudiantes de una población de 208 de acuerdo a la tabla número 6.

Tabla 6 Relación de muestreo aplicado en la Licenciatura de Ingeniería Industrial

\begin{tabular}{|c|c|c|}
\hline Semestre y grupo & Turno & Muestreo \\
\hline $1--1$ & Matutino & 9 \\
\hline $2--1$ & Matutino & 8 \\
\hline $2--2$ & Vespertino & 8 \\
\hline $3--1$ & Matutino & 7 \\
\hline $4--1$ & Matutino & 7 \\
\hline $4--2$ & Vespertino & 7 \\
\hline $5--1$ & Vespertino & 4 \\
\hline $6--1$ & Matutino & 2 \\
\hline $7--1$ & Matutino & 2 \\
\hline $8--1$ & Vespertino & 2 \\
\hline $9--1$ & Vespertino & 2 \\
\hline Total de cuestionarios & 58 \\
\hline
\end{tabular}

Se puede observar que el mayor número de encuestas aplicadas fueron en los primeros 5 semestres de la carrera, ya que son en los que más se presenta el mayor índice de deserción escolar y en base a esta información se realizó la asignación para obtener el tamaño de la muestra. Para aplicar la encuesta en esta licenciatura, se solicitó el permiso de la institución.

Existen artículos recientes realizados sobre deserción escolar donde se mide la confiabilidad y validez. Ventura, Lobos y Gutiérrez [8] dicen que la construcción, validación y confiabilidad de una escala para medir la deserción estudiantil universitaria (CAVU) en la unión de instrumentos aplicados como la deserción estudiantil universitaria y el cuestionario de adaptación a la vida universitaria posee adecuados índices de validez de contenido para medir la deserción.

En otro estudio, Baeza, MJ, Antivio, A y Rehbeien,L [9] estimaron la confiabilidad de una escala de 67 ítems mediante el procedimiento Alpha Cronbach que abarcaron siete factores relacionados con la deserción escolar y resultó favorable superior a 0.7 .

\section{Resultados}

Después de aplicar el cuestionario a los estudiantes en la (LII) de la (UAEH) en la ESTeR para identificar y analizar las principales causas de deserción escolar se tienen 58 respuestas con apreciaciones relevantes.

Los resultados muestran que el $81 \%$ corresponde a estudiantes en un rango de edad entre 17 y 20 años de edad, de los cuales el $62 \%$ son hombres y el $38 \%$ mujeres. Por otra parte, podemos ver que el $41.38 \%$ de los estudiantes se encuentra estudiando en el turno matutino, el $17.24 \%$ por la tarde y en $41.38 \%$ se encuentra en ambos turnos.

Un comportamiento de suma importancia que influye en los índices de deserción de esta licenciatura, es que actualmente los estudiantes compaginan sus estudios con algún tipo de trabajo remunerado, se puede observar que el $31 \%$ de los alumnos actualmente están realizando un trabajo por el cual perciben una remuneración, de los cuales solo el $18 \%$ del trabajo que efectúan está relacionado con sus estudios por lo cual se concluye que estos factores pueden ser motivo de deserción escolar.

Respecto al desempeño académico actual que presenta el $22 \%$ de los estudiantes de la Licenciatura en Ingeniería Industrial ha sido insuficiente por no haber estudiado lo necesario y porque no han sido motivados en las materias. También se puede observar que el $33 \%$ de los estudiantes considera que su desempeño ha sido suficiente y que están siendo motivados con la catedra que están recibiendo en las materias; pero hay un $45 \%$ que están indecisos en base a las dos clasificaciones antes mencionadas dando por hecho que su desempeño la motivación que se tiene de las materias es el suficiente para ellos.

Al revisar la asistencia a clases, se tiene que solo el $6 \%$ de los estudiantes no están asistiendo constantemente a sus clases, aun cuando es un porcentaje bajo no se debe menospreciar ya que lo ideal es tener el $0 \%$ de inasistencias. En relación a la enseñanza, en particular al plan de estudios, los planes docentes de las asignaturas y el contenido de las clases, el $7 \%$ de los estudiantes opinan que no tienen una estructura coherente. Por otra parte, el $22 \%$ opina que estos elementos son suficientes para ellos, pero existe algún nivel de inconformidad el cual hace que los elementos antes mencionados pierdan coherencia en cierto grado.

En cuanto a la relación de las tutorías que han recibido los estudiantes hasta el día de hoy, el 14\% considera que no han 
sido de ayuda para programar el itinerario curricular y planificar su proceso de aprendizaje.

El $26 \%$ de los estudiantes de la licenciatura en Ingeniería Industrial frecuentemente se sienten agobiados(as) sólo de pensar que deben asistir otro día más a clases, esto los hace sentir emocionalmente agotados(as) para cursar su enseñanza.

El $10 \%$ de los estudiantes consideran que el sistema educativo (plan de estudios, proceso de aprendizaje, métodos de evaluación, etc.) no ha sido el adecuado.

En correlación con las expectativas futuras de los estudiantes de ingeniería industrial el $25 \%$ algunas veces se han sentido inseguros (as) sobre si continuar con sus estudios y han considerado la idea de cambiar sus estudios. Los estudiantes de ingeniería industrial han pensado frecuentemente dejar de estudiar para conseguir trabajo, aunque sea mal remunerado en un $17 \%$.

El $11 \%$ opinan que el principal motivo por el cual han pensado dejar los estudios que están cursando ha sido porque piensan que se trata de estudios demasiado difíciles y no podrán desenvolverse como ellos lo quisieran, mientras el $9 \%$ se encuentran indecisos.

\section{Conclusiones}

De acuerdo con el análisis de los datos obtenidos, la presente investigación logró determinar que las causas de deserción escolar de los alumnos de la (LII) en la ESTeR, gracias a la aplicación de un cuestionario validado por la herramienta de Alfa de Cronbach y con una escala de Likert de 5 puntos que dio un coeficiente apto para obtener resultados válidos, donde se pueden visualizar algunas de las posibles causas que describen el comportamiento de la deserción escolar que presenta la (LII) en los últimos 4 años, cabe mencionar que sería importante poder nutrir mucho más esta investigación integrando un análisis y una evaluación en torno a las causas mencionadas en los resultados, a fin de clasificarlas, e identificar cual causa de deserción es la que más impacta en la (LII) y, a su vez conocer el comportamiento de las variables que explicarían el fenómeno para poner en marcha un plan que disminuya los índices de deserción estudiantil.

La aportación principal del estudio es tener un panorama amplio sobre las causas de deserción escolar para que se tomen acciones oportunas, probablemente desde el curso de inducción que se da a los alumnos, una orientación vocacional oportuna, apoyo de los maestros y tutores para orientar a los alumnos en sus decisiones considerando habilidades, destrezas, costos, distancias a las universidades, contenidos de programa entre otras causas.

Comparando con otros estudios de deserción escolar en universidades nacionales se encuentra presente el factor económico la falta de interés por la carrera porque no es lo que esperaban, la falta de organización para trabajar y estudiar. En Este sentido el gobierno ha otorgado becas económicas a los estudiantes, pero falta generar estrategias que permitan disminuir el índice de deserción escolar.

\section{Referencias}

[1] UAEH. (s.f.). Escuela Superior de Tepeji del Río. Obtenido de https://www.uaeh.edu.mx/campus/tepeji/antecedentes.html.

[2] UAEH. (2019). Información para el estudio de Trayectorias Escolares. Obtenido de http://sgc.uaeh.edu.mx/planeacion/index.php/estainst?id=54

[3] OCDE (2010). Organización para la cooperación y desarrollo económicos. Acuerdo de cooperación México- OCDE para mejorar la calidad de la educación de las escuelas mexicanas disponible en http//: www.oecd.org

[4] EDURAMA (2019). Deserción Universitaria: los datos en México. Disponible en revista digital http//: www. edurama.com.

[5] INEGI. Instituto Nacional de Estadística Geografía e Informática (2018). Disponible en http: //www.inegi.com.mx

[6] MOLINA, J. A. (2013). Utilización del alfa de Cronbach para validar la confiabilidad de un instrumento de medición de satisfacción del estudiante en el uso del software Minitab. LACCEI, 9.

[7] BELCHER, W. L. (2010). Cómo escribir un artículo académico en 12 semanas . MÉXICO: FLACSO MÉXICO.BELCHER, W. L. (2010). Cómo escribir un artículo académico en 12 semanas . MÉXICO: FLACSO MÉXICO.

[8] VENTURA, JM, LOBOS, ME Y GUTIÉRREZ, JR. (2019). Construcción, validación y confiabilidad de escala de medición de deserción estudiantil universitaria. Entorno, junio 2019, número 67.

[9] BAEZA, MJ; ANTIVIO, A. Y REHBEIN, E.L. (2016)

Diseño y validación de una escala de Preparatividad académica para la educacion superior en Chile Formación Universitaria Vol 9(4) 63-74. 